Noname manuscript No.

(will be inserted by the editor)

\title{
Real-time droplet caliper for digital microfluidics
}

\author{
Matthieu Robert de Saint Vincent • \\ Sébastien Cassagnère • Joël Plantard • \\ Jean-Pierre Delville
}

the date of receipt and acceptance should be inserted later

\begin{abstract}
We developed an optical, microfabrication-free, approach for performing real-time measurements of individual droplet characteristics (frequency of production, velocity, and length) flowing in a transparent microfluidic channel. Our approach consists in an interpretation of the differential signal produced by a pair of photodiodes connected head-to-tail due to the variations of illumination at the passage of a droplet. We checked the relevance of this zero-background method by comparing results to video measurements, and observed a very good agreement at rates up to the $\mathrm{kHz}$ range. Moreover, since the measured values are stored in a simple text file, flow characterization over very long times (several hours) becomes
\end{abstract} M. R.S.V.

Present address: School of Physics and Astronomy, University of St Andrews, North Haugh, Fife, KY16 9SS, Scotland, UK

M. R.S.V. · S. C. · J. P. and J.-P. D.

Univ. Bordeaux, LOMA, UMR 5798, F-33400 Talence, France.

CNRS, LOMA, UMR 5798, F-33400 Talence, France,

E-mail: j.plantard@loma.u-bordeaux1.fr, E-mail: jp.delville@loma.u-bordeaux1.fr 
accessible. We applied this facility to perform three examples of long-term studies: stationary regimes, transient regimes, and the effect of an external forcing. Several unexpected features, like long-period fluctuations, can thus be evidenced.

Keywords digital microfluidics · flow characterization · measurement in fluid dynamics $\cdot$ real-time characterization $\cdot$ transient regimes

\section{Introduction}

Over the past decade, droplet microfluidics (Teh et al 2008) also referred as digital microfluidics, has raised a growing interest in the scope of lab-on-a-chip applications. This interest is motivated by the fact that droplets flowing in a carrier fluid are very good candidates for performing (bio-)chemical reactions since the immiscibility prevents from any cross-contamination (Song et al 2006; Huebner et al 2008). Furthermore, this approach allows for performing multiple reactions, either in series or in parallel, on the same device (Dittrich et al 2006; Trivedi et al 2010). Several methods for producing droplets at high rates (up to the $\mathrm{kHz}$ range), with well-defined size distribution, have been proposed (Christopher and Anna 2007), and the study of two-phase flows remains an active field of research (Lee et al 2009). To measure the characteristics (size, velocity, and frequency of production) of droplets, the most popular method consists in analyzing video frames recorded with a fast camera. While this procedure allows for an exhaustive characterization of a droplet population, it exhibits two inherent limitations. First, recording over very long times (beyond a few minutes), which can be required to investigate transient flows of very viscous fluids, needs for unreasonable data storage facilities. Second, the analysis of video frames is time-consuming and, even more important, cannot be performed in real 
time, as a further processing of the video frames is required. This point is also of crucial relevance since many microfluidic applications require to stand in a stationary regime. As the characteristic time of transient regimes can vary over several orders of magnitude, depending on viscosity contrast, fine adjustments of the flow rates to specifically fit with the application requirements can indeed be quite challenging.

Despite these limitations, relatively few alternatives to the analysis of $2 \mathrm{D}$ video frames were proposed for measuring droplets in digital microfluidics, in contrast with the large variety of detection tools, for example, for chemical analysis (Götz and Karst 2007; Baker et al 2009). Very recently, Jakiela et al (2011) used a linear camera imaging the centerline of the channel only to prevent excess data storage, reduce data processing by simply detecting the front and back droplet interface, and thus perform real-time investigations of droplet velocity and length. Nevertheless, most non-imaging techniques are mainly optical. They are based on the variation of illumination during droplet flow. The most wide-spread setup consists of a single photodiode placed on the axis of a laser beam crossing the channel. Such a device allows for real-time droplet detection (Engl et al 2005) but cannot achieve any measurement on these droplets, except the frequency of droplet production. Nguyen et al (2006) integrated an optical fiber in a microfluidic chip, and observed that the signal shape depends on the droplet size. However, neither the size nor the velocity of the droplets are quantitatively measured since the signal shape is not associated with any length of reference. Extending the method to two fibers separated by a well-defined distance, Revellin et al (2006) cross-correlated temporally the two independent signals to get the mean velocity of droplets and deduced their length when longer than the fiber inter-distance (slugs). However, the absence of length of reference still forced 
these authors to adapt signal thresholds from experiment to experiment to measure the slug size.

A more sophisticated alternative, proposed for larger scale devices, is to use optical fibers themselves as probes of the flow. A single fiber, placed on the droplet path, pierces the fluid interface, and the signal detected during the interface-probe interaction allows for evaluating both droplet length and velocity (Hironaga and Nishiura 1985; Cartellier 1990, 1992; Lim et al 2008). In addition to the complexity of the setup, this intrusive method introduces errors in measurement since the probe modifies the droplet shape and the flow velocity. A less invasive variant of this method involves several probes placed perpendicularly to the flow (de Lasa et al 1987), but the setup remains complex and rigid.

Recently, Cox and Zins (2006, 2010) patented a non-invasive optical approach for measuring small objects like blood cells. The projected shadow of these objects is detected successively by a pair of photodiodes, giving access to their mean velocity. Furthermore, as far as the objects are small compared to the sensors, the amplitude of the signal depends on their size, which can thus be determined providing that the device has been calibrated with a flow of particles of known size. This relatively simple method suits well for measuring small monodisperse particles (cells, typically) but is limited to the micrometer range and moderate flow rates, since the objects must be detected one by one.

In addition to these optical approaches, we should mention alternatives based on the modification of the electrical properties of the investigated volume of fluid due to the presence of a droplet. Resistive methods (Sun and Morgan 2010) are widely employed in cytometry. Also, Niu et al (2007) developed a capacitive measurement, which detects the passage of a droplet and determines, after an appropriate signal 
processing, its velocity, and less confidently its length due to signal convolution.

Moreover, Liu et al (2008) used chronoamperometry to measure the droplet frequency and size, providing that a preliminary optical calibration is performed. An extension to a dual electrode setup, in order to access to the velocity, was also presented. An electrolyte was nevertheless required and the method is limited to low flow rates. Note finally that all these electrical methods need the integration of electrodes within the channel, and then dedicated microfabrication.

We propose in this paper a simple, microfabrication-free, optical method to measure in real time all the characteristics of droplets in digital microfluidics. This method is based on the differential detection of the variations of illumination of the projected shadow of the flowing droplet onto a pair of close photodiodes connected head-to-tail.

\section{Principle of the measurement}

The whole setup used to validate the method is represented in Fig. 1. The key elements are a microscope (here, an Olympus IX 71, with a $\times 5$, N. A. 0.15 objective, but a much simpler one is sufficient), the electronic measuring device (hereafter designed as "detector"), and a computer. The detector consists of two identical commercially available bare photodiodes, with a sensitive surface of $15 \mathrm{~mm} \times 100 \mu \mathrm{m}$, on which the image of water droplets carried by an oil flow in a transparent microchannel is projected, the axis of flow being perpendicular to the photodiodes. The photodiodes are electrically connected head-to-tail (i.e., anode $i$ is connected to cathode $j$, with $i \neq j=1,2$, as depicted on Fig. 1). Currently, the chosen photodiodes are separated by a $370 \mu \mathrm{m}$ interspacing which corresponds to the case where the substrate of the 
photodiodes are in physical contact. The resulting signal, called "differential current" $I_{d}$, thus corresponds directly to the difference between the current $I_{1}$ and $I_{2}$ produced by each photodiode, contrary to a classical scheme where two independent signals would be further processed. Here the signal corresponds to an imbalance in illumination between the two sensors, allowing for the detection of very weak variations while eliminating the effect of the ambient noise. The differential current is then converted into voltage and amplified, and an offset is added to adjust the baseline. The interpretation of the signal is based on the detection of threshold levels (see below), defining logic signals, which are transmitted to a computer via an USB connection. These signals are processed by a program written in LabviEw ${ }^{\mathrm{TM}}$ to deduce the characteristics of successive droplets. The corresponding data are displayed, and stored in a text file in real time. A detector composed of two identical and close photodiodes separated by a small gap is essential from many regards: (i) to give access to the instantaneous droplet velocity, which is the only valuable quantity in any situation as droplet velocity in channels is known to depend on the traffic and then may vary continuously during displacement; (ii) to confidently deduce the droplet length from its instantaneous velocity and the time span by its moving image over the detector length from signal thresholds defined once and for all and, (iii) to tremendously increase the signal-to-noise ratio by head-to-tail connection since electronic offsets, and physical and environmental noises are automatically eliminated.

To validate our setup, we compared results given by the detector to simultaneous measurements obtained from further video analysis. To this aim, a beam splitter was used to duplicate flow visualization on a CMOS camera. Moreover, an oscilloscope was connected to the detector to get in real time both analog and logic signals. 


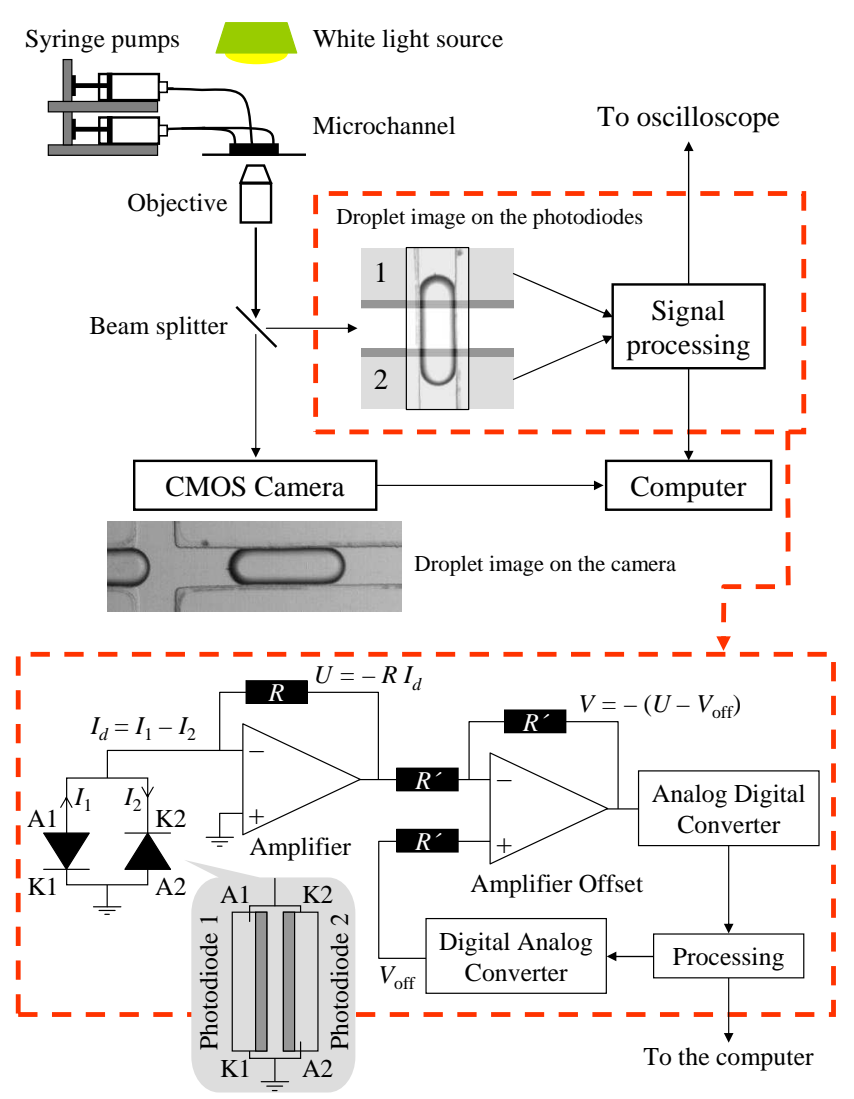

Fig. 1 Schematics of the setup. The detector is detailed in the dashed box.

The microchannel is cross-shaped, of width $w \simeq 100 \mu \mathrm{m}$ and height $h \simeq 50 \mu \mathrm{m}$, molded in PDMS by standard soft lithography techniques (Duffy et al 1998), and sealed on a glass slide. The used fluids are basically millipore water and hexadecane (hereafter called "oil"), with the addition of the widely-used Span 80 surfactant ( $2 \% \mathrm{wt}$ ). Water and oil are set in motion by syringe pumps (alternatively, gravitydriven flows can be performed by suspending open reservoirs), thus leading to the formation of water droplets near the cross region, as represented in the picture of Fig. 1. Both fluids are transparent, but droplet edge appears dark since the microscope 
illumination is totally reflected in oil (refractive indexes of water and oil are 1.33 and 1.43 , respectively).

Let us now consider the signal shape as a droplet image crosses the pair of photodiodes. In Fig. 2 are plotted together oscilloscope traces, in light black line, and the signal corresponding to the integration of gray levels over the apparent sensing surfaces on video frames, in bold red line. The very good overlap of these two traces confirms the relevance of our interpretation of the signal in terms of detection of projected drop shadow onto the photodiodes.

The case of a droplet image larger than the interspacing between the photodiodes is represented in Fig. 2 (a). When the front interface starts to hide photodiode 1, it detects a much lower light intensity than the other photodiode, resulting in a strongly imbalanced, negative signal (step i). The signal reaches a first minimum when the projected area of the shadow on photodiode 1 is maximal. When the droplet front is located between the two photodiodes, the signal remains negative but decreases (in absolute value) as the shadowed area decreases (step ii). Then the droplet front reaches photodiode 2, which therefore detects a decreasing intensity. The shadowed area on photodiode 2 eventually becomes larger than on photodiode 1 , and the signal becomes positive (step iii), up to a first maximum. This maximum is always smaller than the absolute value of the first minimum since both photodiodes are partly overlapped by droplet edge. The signal then nullifies as both photodiodes are equivalently shadowed (step iv). The rear face of the droplet finally induces a complementary and reverted signal (steps v and vi), defining a second minimum and a second maximum, successively.

Note that the amplitude of the first minimum and of the last maximum only depend on the shape of front and rear faces of the droplet. For confined droplets, 
(a)
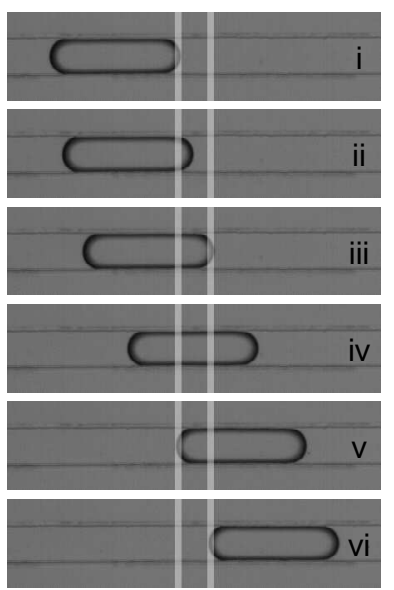

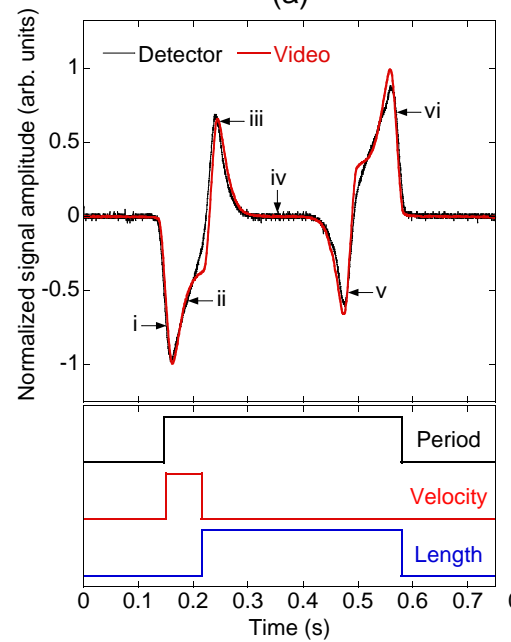

(b)

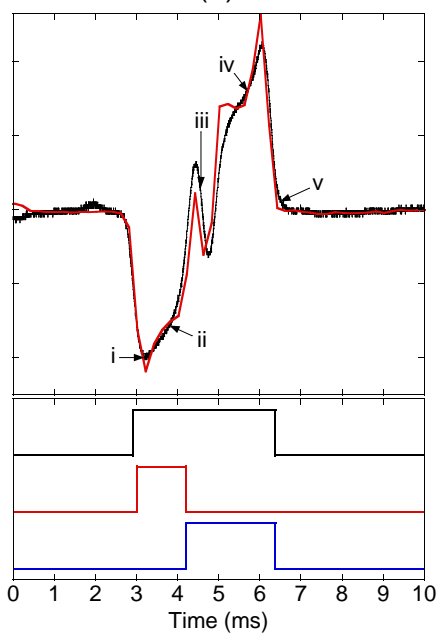

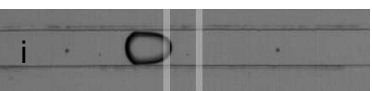
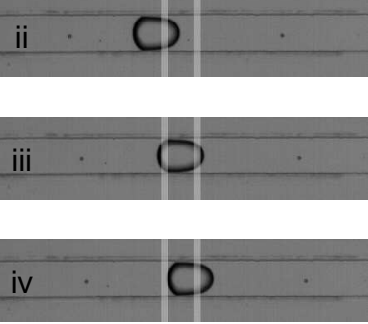

V

Fig. 2 Signal obtained as a droplet image crosses the photodiodes, for two couples of flow rates:

(a) oil, $Q_{o}=0.4 \mu \mathrm{Lmin}^{-1}$, and water, $Q_{w}=0.1 \mu \mathrm{L} \mathrm{min}^{-1}$, (b) $Q_{o}=25 \mu \mathrm{L} \mathrm{min}^{-1}$ and $Q_{w}=$ $2.5 \mu \mathrm{L} \mathrm{min}^{-1}$. Upper graphs represent the analog signal, as plotted by the oscilloscope (light black traces), and as measured on the video frames by gray level detection (bold red traces). Lower graphs represent the logic signals resulting from the threshold detection for measuring the droplet period, velocity, and length (see text). Left and right panels are representative frames (as projected on the detector) illustrating the main steps of the shadow measurement, for cases (a) and (b), respectively. The sensitive part of the two photodiodes have been depicted in light gray. The channel width is $100 \mu \mathrm{m}$. (See electronic supplementary material for a dynamic view of the full processing).

in the limit of low capillary numbers, this shape is imposed by the lateral walls, and does not depend on droplet size and velocity. Therefore, the first minimum can be considered as a reference value for measuring the droplet size and velocity, whatever further possible evolution of the flow. Note finally that the size of the droplets produced in the channel is not a hurdle since one can easily change the magnification of the microscope objective used to image droplets on the detector.

The technique is thus generalizable from a few microns to the millimetric range. 
The case of shorter droplets, of length comparable to the apparent distance between the two photodiodes, is considered in Fig. 2 (b). The signal shape is similar to the large droplet case, but the amplitude of intermediate extrema is smaller. This is due to the simultaneous overlap of the two photodiodes signals due to the front and rear curved faces (see image iii). We also notice that in this case, the two faces of the droplet have different shapes since its velocity (and therefore the capillary number) is larger than in the previous case. However, it does not significantly influence the signal shape.

The signal being well understood, we can easily determine when a droplet front reaches a specific location. Therefore, we define three logic signals, as represented in the lower graphs of Fig. 2, corresponding to the time necessary for the droplet to cover a known distance. Indeed, determining the extreme values of the signal is difficult to achieve precisely in real time, so they are determined on the droplet preceding the droplet to be measured, and progressively updated. However, we recall that these values do not significantly change from one drop to another, as far as their shape is imposed by channel walls. A first threshold $\left(t_{50}\right)$ is defined when the signal reaches $50 \%$ of the previous first minimum. Two successive thresholds $t_{75}^{(1)}$ and $t_{25}^{(1)}$, respectively, are then associated to negative and positive slopes. Two additional thresholds, $t_{75}^{(2)}$ and $t_{25}^{(2)}$, are finally determined at 75 and $25 \%$ of the last maximum. Note that the choice of these threshold values aims at optimizing the speed of processing, but different values can be chosen as well. The only requirement is to know the position of the droplet front which is associated to these values.

The signal "period" starts at $t_{50}$, and arbitrarily stops at $t_{25}^{(2)}$. This signal is a counter, indicating at what time a droplet is detected. The time delay between two 
successive droplets, or period, is therefore the time delay between two successive $t_{50}$ 's.

The signal "velocity" starts at $t_{75}^{(1)}$ and stops at $t_{25}^{(1)}$. During this time, the droplet covers the distance separating the two photodiodes. We can thus deduce the velocity of the droplet as it crosses the detector.

When velocity is known, determining the droplet length would require to detect at the same point the front and rear interfaces successively, i.e., for example, between steps i and v, or iii and vi [Fig. 2 (a)]. However, this procedure involves the intermediate maximum, which depends on droplet size. To circumvent this difficulty, we rather consider the time necessary for the droplet to cover its length plus the width of the sensing zone of photodiode 2 . The signal "length" is thus defined between $t_{25}^{(1)}$ and $t_{25}^{(2)}$.

Considering these three signals, we are thus able to fully characterize a train of successive droplets flowing in the channel. Supplementary material also presents a video that sums up and dynamically illustrates the full process. Let us now compare these measurements to those deduced from the video analysis.

\section{Validation of the method}

\subsection{Comparison for different flow rates}

Figure 3 represents the period, velocity, and length results, in the case of water-in-oil droplets. Measurements performed by the two methods are compared in Fig. 3 (a) for moderate flow rates (oil, $Q_{o}=0.4 \mu \mathrm{L} \mathrm{min}^{-1}$, and water, $Q_{w}=0.1 \mu \mathrm{L} \mathrm{min}^{-1}$ ). In this case, the length of the projected image of the droplet onto the setup is larger than the distance between the photodiodes. The velocity and frequency of emission 
(a)

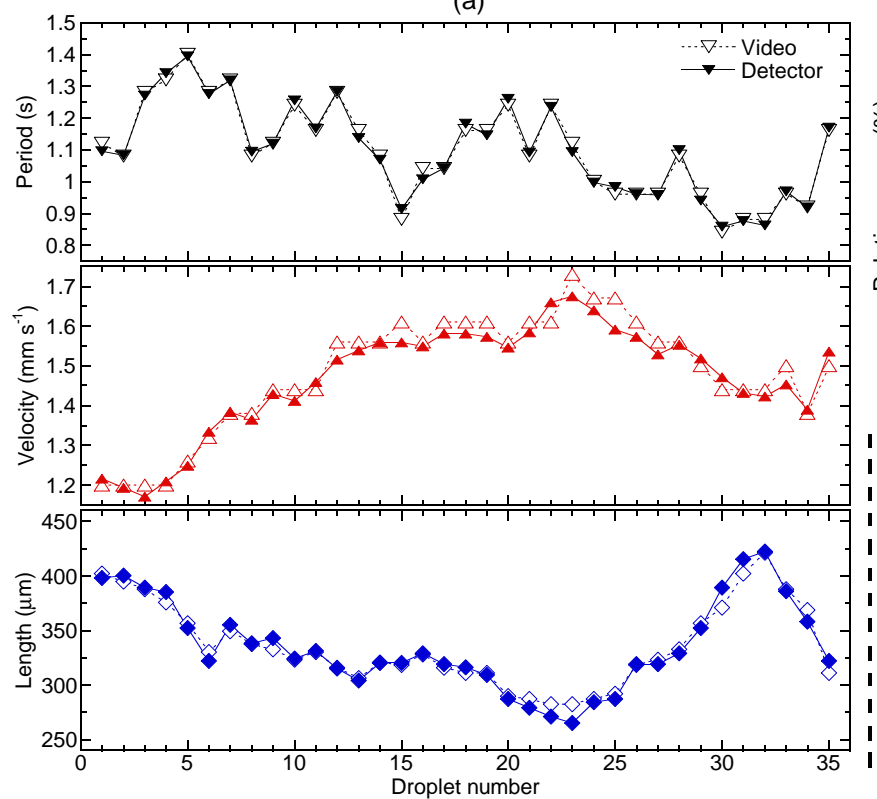

(b)

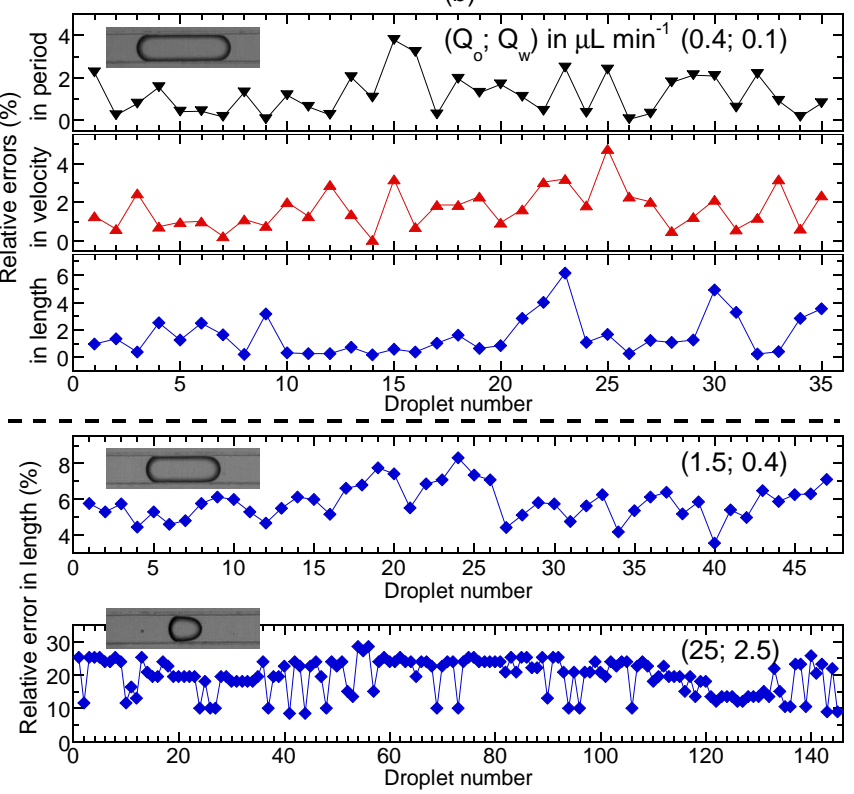

Fig. 3 (a) Comparison between the period, velocity, and length of successive droplets measured by the detector (filled symbols) and deduced from the video analysis (open symbols). Flow rates are $Q_{o}=0.4 \mu \mathrm{L} \mathrm{min}^{-1}$ and $Q_{w}=0.1 \mu \mathrm{L} \mathrm{min}^{-1}$. (b) Relative error in the detector measurements. From top to bottom, errors in period, velocity, and length at moderate flow rates, and errors in length at intermediate and high flow rates. Channel width in images is $100 \mu \mathrm{m}$.

are in the $\mathrm{mm} \mathrm{s}^{-1}$ and $\mathrm{Hz}$ ranges, respectively. Each droplet can be identified from the fluctuations on droplet characteristics at the individual level, allowing for a direct comparison between both types of characterization. We can observe that both video analysis (open symbols) and detector measurements (filled symbols) yield very close value for each physical quantity of interest. The error is typically below $4 \%$ within these conditions, as can be seen on the upper graphs of Fig. 3 (b).

At intermediate flow rates $\left(Q_{o}=1.5 \mu \mathrm{L} \mathrm{min}^{-1}, Q_{w}=0.4 \mu \mathrm{L} \mathrm{min}^{-1}\right)$, the projected droplet image is still larger than the photodiode interspacing. Droplet velocity 
and frequency of emission are about $5 \mathrm{mms}^{-1}$ and $5 \mathrm{~Hz}$, respectively. The fluctuations of droplet characteristics still allow for direct video/detector comparison (not shown). Both measurements share the same trend, but the detector systematically underestimates droplet velocity and length. This results in a systematic error of about $6 \%$ in the measured length, as represented in the middle graph of Fig. 3 (b).

High flow rates $\left(Q_{o}=25 \mu \mathrm{L} \mathrm{min}^{-1}\right.$ and $\left.Q_{w}=2.5 \mu \mathrm{L} \mathrm{min}^{-1}\right)$ lead to the formation, at a frequency close to $70 \mathrm{~Hz}$, of very regular droplets, flowing at a velocity close to $7 \mathrm{~cm} \mathrm{~s}^{-1}$. In this case, the droplet length image is comparable to the distance between the photodiodes for the chosen objective magnification [see Fig. 2 (b)]. Since the fluctuations in period and velocity are too weak to be detected by the camera at the operating frame rate, we were not able to compare each droplet measurement individually. Nevertheless, the length determined by the detector remains systematically below that measured on the video frames by about $25 \%$, as represented in the lower graph of Fig. 3 (b).

We finally tested the case (not shown) of very high flow rates $\left(Q_{o}=88 \mu \mathrm{L} \mathrm{min}{ }^{-1}\right.$, the maximum value allowed by the syringe pump, and $Q_{w}=10 \mu \mathrm{L} \mathrm{min}^{-1}$ ). Droplets are $100 \mu \mathrm{m}$ large, the frequency of emission is about $0.5 \mathrm{kHz}$, and their velocity is $28 \mathrm{~cm} \mathrm{~s}^{-1}$. All droplets are detected, but their velocity and length are included in an interval defined by two digitized values $\left(24\right.$ and $35 \mathrm{~cm} \mathrm{~s}^{-1}$, and 75 and $160 \mu \mathrm{m}$, respectively). The agreement remains much better than the order of magnitude, and the fact that all droplets are detected demonstrates that the principle of the measurement remains relevant in the $\mathrm{kHz}$ range. The present quantitative limitation can thus be overtaken by modifying the electronic components.

All the comparisons performed here show that the detector gives very accurate measurements (error is typically below 10\%) up to the $\mathrm{cms}^{-1}$ range. For faster 
droplets, the actual device must be further optimized in the sense that electronic components can easily operate at the $\mathrm{kHz}$ range.

\subsection{Comparison for different refractive indexes}

The principle of the measurement is based on the detection of shadows due to a contrast in refractive index between the two phases, $\Delta n=n_{w}-n_{o}$. In the waterhexadecane case, $\Delta n=-0.10$, the minus sign denoting a possible total internal reflection of the microscope illumination at the oil-water interface. To demonstrate the generality of the method, we also considered two different dispersed phases: (i) Argon bubbles, and (ii) water droplets containing sucrose. In the case of gas bubbles, the contrast in refractive index, still negative, is strengthened $(\Delta n=-0.43)$. Bubbles therefore present a thick dark edge, as can be seen in the left image of Fig 4 (a). For droplets of sweetened water, the concentration in sucrose $(60 \%$ wt) was chosen for setting the refractive index of the solution very close to that of hexadecane $(\Delta n<0.01)$. The residual mismatch in $\Delta n$ is imposed positive to ensure light to be always refracted. On video frames, we were able to make out droplet edge, which does not appear dark. In the right image of Fig. 4 (a), the front and the rear faces of a syrup droplet are depicted by the arrows.

Representative oscilloscope traces are also represented in Fig. 4 (a), for gas bubbles (left) and syrup droplets (right). The signal shape is very similar to the waterhexadecane case discussed above (see Fig. 2), and the good overlap between the oscilloscope trace (light black line) and the gray level integration (bold red line) is also retrieved here. Evidently, the signal is much more noisy in the case of sweetened water. 
(a)
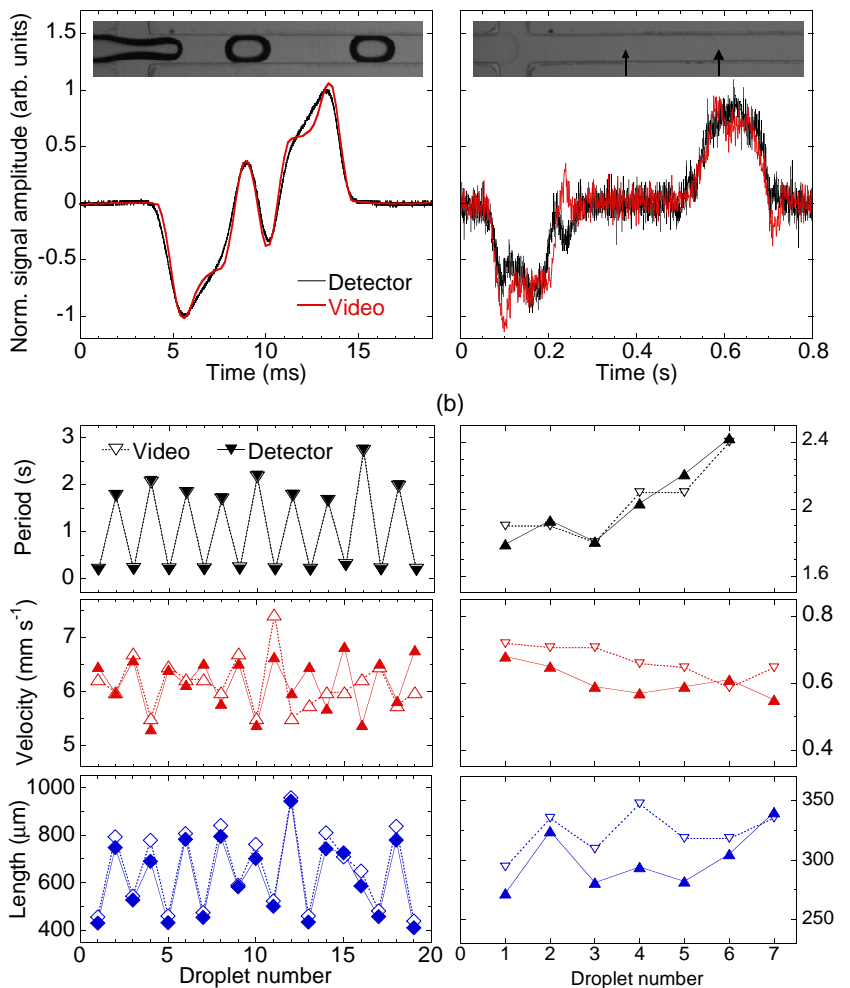

Fig. 4 (a) Oscilloscope traces (light black) and gray-level integration (bold red) obtained in the case of gas bubbles (left) and 60\%-sucrose water droplets (right) flowing in hexadecane, as represented on the frames in insets. The two arrows on the right image indicate the position of droplet front and rear. (b) Comparison of the period, velocity, and length obtained from detector (filled symbols) with those given by video analysis (open symbols), for gas bubbles (left) and sweetened water droplets (right).

Figure 4 (b) compares the measurements obtained by the detector to those given by the video analysis. Gas bubbles (left panel) were produced by using a pressurized bottle connected to the channel by a pressure regulator. At the imposed oil flow rate,

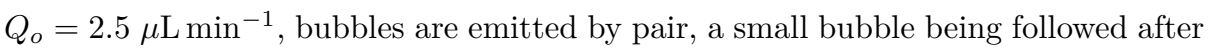
$\sim 0.2 \mathrm{~s}$ by a larger one. Then the gas thread retracts, and another pair is emitted after $2 \mathrm{~s}$. All bubbles flow at a velocity of the order of $6 \mathrm{~mm} \mathrm{~s}^{-1}$. The detector succeeds 
in detecting, and measuring alternatively both large and short bubbles, with errors typically below $10 \%$ (length) to $15 \%$ (velocity). This error range is comparable to that of water droplets emitted in similar conditions [intermediate case in Fig. 3 (b)], suggesting that increasing the signal-to-noise ratio by a higher contrast does not significantly improve the accuracy of threshold detection.

Droplets of sweetened water (Figure 4 (b), right panel) are emitted every $\sim 2 \mathrm{~s}$, and flow at a velocity close to $0.6 \mathrm{~mm} \mathrm{~s}^{-1}$ (imposed flow rates: $Q_{o}=0.2 \mu \mathrm{L} \mathrm{min}^{-1}$ and $Q_{w}=0.05 \mu \mathrm{L} \mathrm{min}^{-1}$ ). In these conditions, comparable to the most favorable flowing conditions studied with pure water droplets (moderate flow rates in Fig. 3), typical error is smaller than $15 \%$. However, the agreement in trend suggests that the interpretation of differential signal remains valid even with very poorly contrasted interfaces.

In the present section we have demonstrated the ability of our differential signal interpretation to fully characterize two-phase flows at frequencies up to the $\mathrm{kHz}$ range, and velocities up to several tens of $\mathrm{cms}^{-1}$, even in the case of poorly contrasted interfaces. Moreover, the ability to discriminate populations of different sizes has been demonstrated as far as droplet size remains larger than the channel width. The present signal interpretation is mainly limited by the fact that the distance separating two successive droplets must be at least equal to the sensor total width. This limitation can be partly overcome by using an objective of stronger magnification.

\section{Applications}

The potentialities of the method open the way to various perspectives in two-phase flow studies. We present in this section three examples of such measurements. 
4.1 Stationarity of imposed flows

As stated above, stationary regimes are of particular relevance for both applied and fundamental microfluidic studies. However, do constant imposed flow rates inevitably lead to stationary regimes? Over short times, irregular fluctuations on droplet individual characteristics [see, e.g., Fig. 3 (a)] can be seen through video analysis, especially at moderate flow rates. Periodic fluctuations have also been investigated recently over intermediate time scales (several minutes) by Korczyk et al (2011). They observed sawtooth-like fluctuations of about $10 \%$ of the mean droplet volume when produced by syringe pump and almost no fluctuations for pressurized feeding. To generalize these statements, we measured droplet frequency of emission, velocity, and length, over a much longer time - one night, to reduce as much as possible the surrounding noise; during this time up to 250,000 droplets were detected - by varying the conditions of injection (syringe pumps, flow rates, or pressure). Note that the setup is mounted on a floating table and that air conditioning was shut down during the measurements. Results are plotted in Fig. 5.

The data represented in Fig. 5 (a) and (b) correspond to a flow imposed at constant rates, $Q_{o}=1.5 \mu \mathrm{Lmin}^{-1}$ and $Q_{w}=0.4 \mu \mathrm{L} \mathrm{min}^{-1}$, by a pair of syringe pumps KD Scientific KDS 100 (a) or Harvard Apparatus PHD 2000 (b). Two main features have to be mentioned. First, the mean characteristics of the two populations are different while the imposed flow rates are the same. This could be attributed to differences in calibration between the two devices (the same microchannel and pipes were used for all experiments), or to slight differences in material properties, which can result from fluctuations in ambient temperature from one day to another. Second, a remarkable succession of periodic events can be observed in both case. Frequency 
(a)

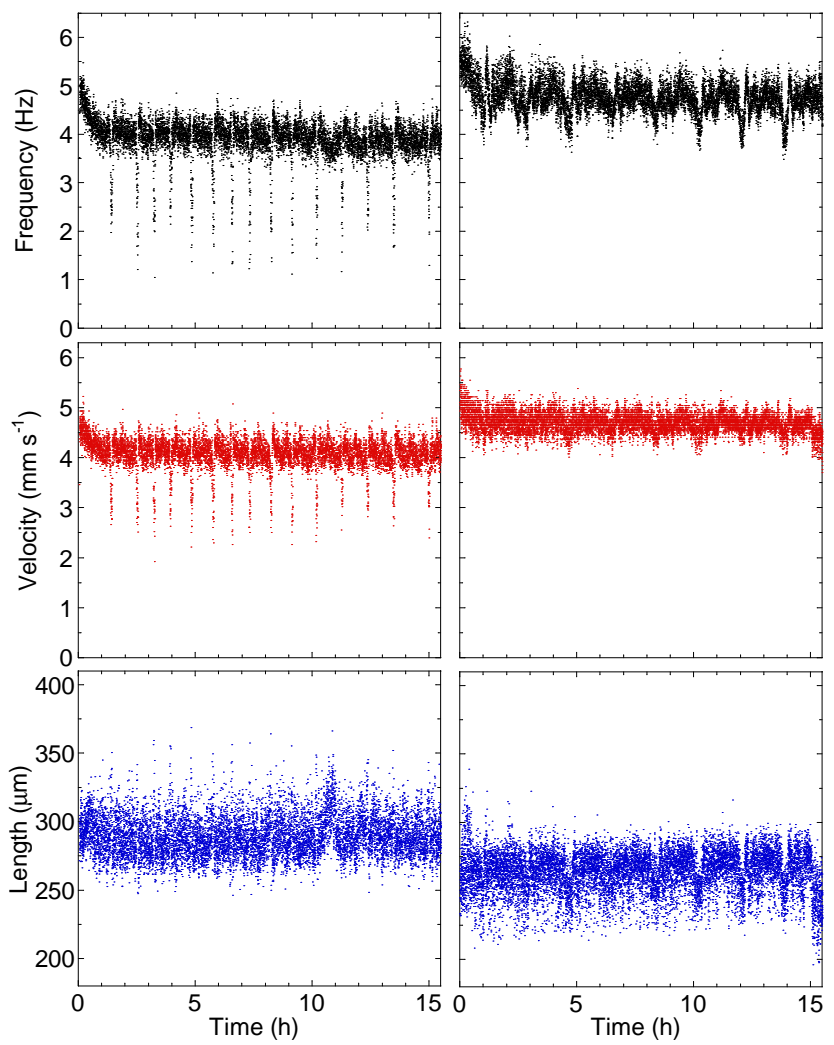

(c)

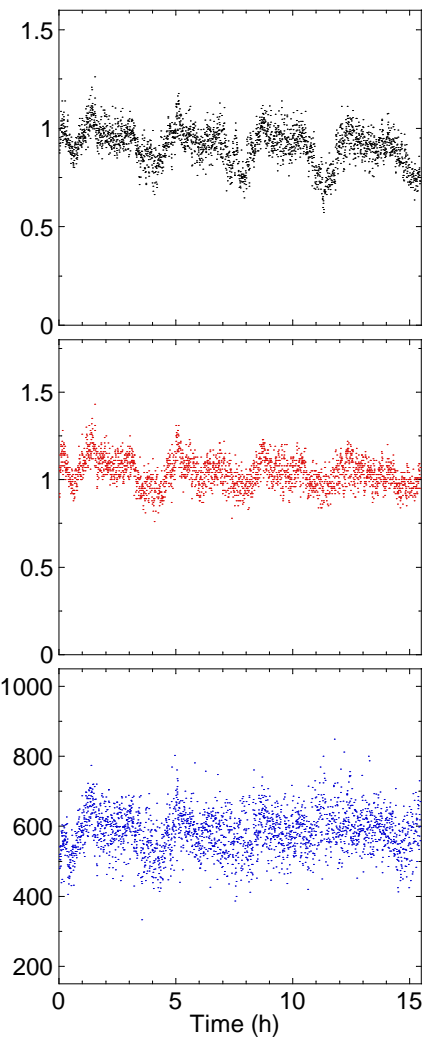

(d)

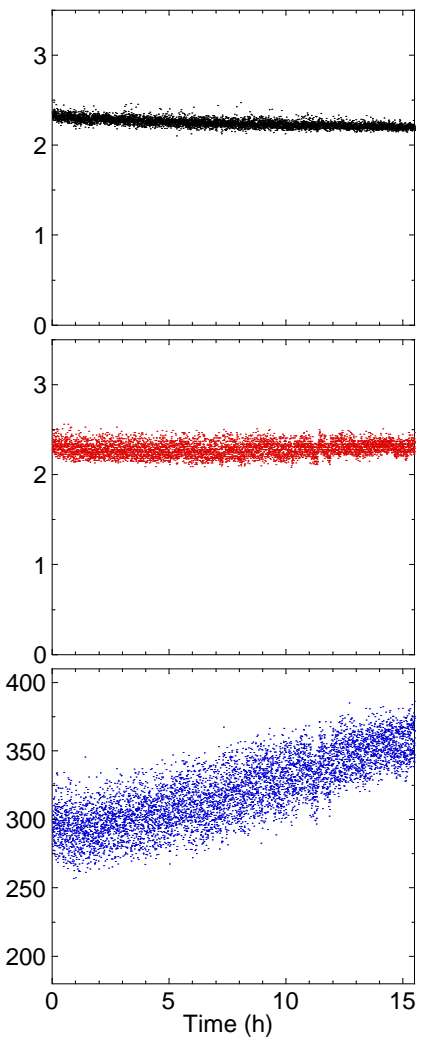

Fig. 5 Measured frequency of emission, velocity, and length, of droplets produced at imposed flow rates (a-c) or pressures (d). Syringe pumps: (a) KDS 100, and (b,c) Harvard PHD 2000; oil and water flow rates (in $\mu \mathrm{L} \mathrm{min}^{-1}$ ): (a,b) 1.5 and 0.4, (c) 0.3 and 0.2 , respectively. (d) Initially imposed pressures: 2502 (oil) and 2350 (water) Pa.

of emission, and flowing velocity, significantly drop during these events. We can also notice an increase [KD Scientific KDS 100, case (a)] or a decrease [Harvard Apparatus PHD 2000, case (b)] in the length of the corresponding droplets.

Then we operated the Harvard Apparatus PHD 2000 syringe pumps at another couple of smaller imposed flow rates, $Q_{o}=0.3 \mu \mathrm{L} \mathrm{min}^{-1}$ and $Q_{w}=0.2 \mu \mathrm{L} \mathrm{min}^{-1}$ [Fig. 5 (c)]. Complex periodic fluctuations, composed of $\sim 4 \mathrm{~h}$ main cycles with a 
mid-term secondary event, can be observed. The long-term fluctuation patterns thus depend both on the syringe pump used and on the imposed flow rates.

Do pressure-driven flows also exhibit such behavior? To answer this question we also performed measurements with fluids driven at constant pressure by gravity [Fig. 5 (d)]. Oil and water pressures were initially fixed at $\Delta p_{o}=2502 \mathrm{~Pa}$ and $\Delta p_{w}=$ $2350 \mathrm{~Pa}$, respectively. Droplet frequency of emission and velocity are remarkably regular over the whole duration of the experiment (still one night), and do not exhibit any periodic fluctuation. However, droplet length linearly increase as fluids consume. Indeed, after $16 \mathrm{~h}$ the decrease in oil and water levels yields a reduction of pressure by 99 and $49 \mathrm{~Pa}$, respectively. The resulting increase in pressure ratio, $\Delta p_{w} / \Delta p_{o}$, should lead to an increase in droplet size (Ward et al 2005), as observed.

In the absence of periodic variations for pressure-driven flows, any possible fluctuations due to materials elasticity-PDMS of the microfabricated channel, polyethylene of the pipes - cannot be invoked as a reasonable source of this complex behavior. However, as periodicity is inversely related to the flow rates, these periodic fluctuations can rather be ascribed to mechanical effects either due to the mechanics of the syringe pumps, or the solid friction inside the syringe. Data sheets of the syringe pumps are nonetheless not sufficient to go further and get clear-cut insights on possible mechanical drawbacks. Korczyk et al (2011) already discussed these mechanical effects. One can note, however, that their investigation was limited to high flow rates as they cannot perform measurements over time scales of hours. Our method then extends previous observations toward low rates, where both the period and amplitude of fluctuations increase, which are particularly relevant to the applications that require very low flow rates (e.g., microfluidic applications of optical forces (Milne et al 2007; Padgett and Di Leonardo 2011)). 
4.2 Transient flows

Changing the characteristics of the produced droplets is achieved by modifying the conditions of injection. However, reaching the desired characteristics is not instantaneous, and different types of transient regimes may be observed. To the best of our knowledge, very few studies of these transient flowing regimes can be found in the literature (Bong et al 2011) while very long relaxation times can be expected in some cases, like in very viscous or viscoelastic fluids, thus preventing an easy quantitative study from video analysis. We demonstrate the feasibility of such studies by measuring in real time the changes of droplet individual characteristics resulting from a brutal change in either flow rates or pressures, in the case of water droplets in hexadecane. The corresponding measurements are represented in Fig. 6. Starting from a stationary regime, we change at $t=0$ the conditions of injection of both oil and water phase.

Figure $6(\mathrm{a}, \mathrm{b})$ show the evolution of droplet characteristics at imposed flow rates, for both a strong rate increase and the symmetric decrease, respectively. These modifications are expected to be practically instantaneous since the response time of the syringe pumps is less than $1 \mathrm{~s}$. Therefore, the measured dynamics should be associated to the transient hydrodynamic response to these modifications. Droplet period of emission and velocity change by two orders of magnitude, although changes in length are less important. A remarkable feature is that the increase in flow rates and the symmetric decrease do not yield symmetric behaviors. The former leads to steep changes in droplet characteristics, without droplet emission during the first $6 \mathrm{~s}$ [Fig. 6 (a)], whereas droplet period of emission and length (velocity) gradually increase (decreases) during about $30 \mathrm{~s}$ when reducing the flow rates [Fig. 6 (b)]. 
(a)
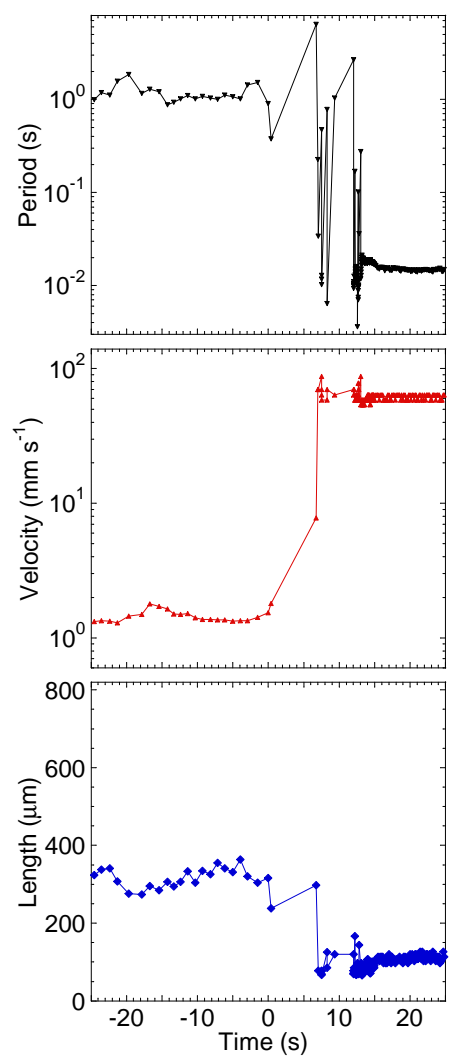

(b)
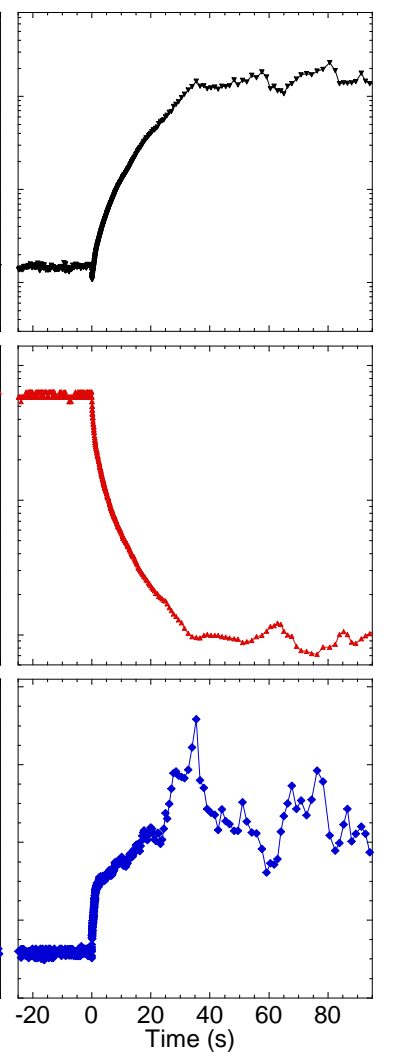

(c)
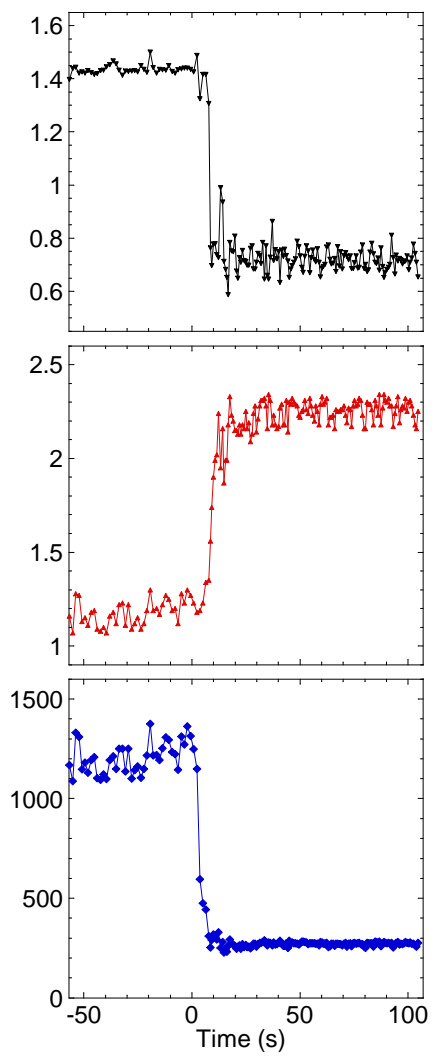

(d)

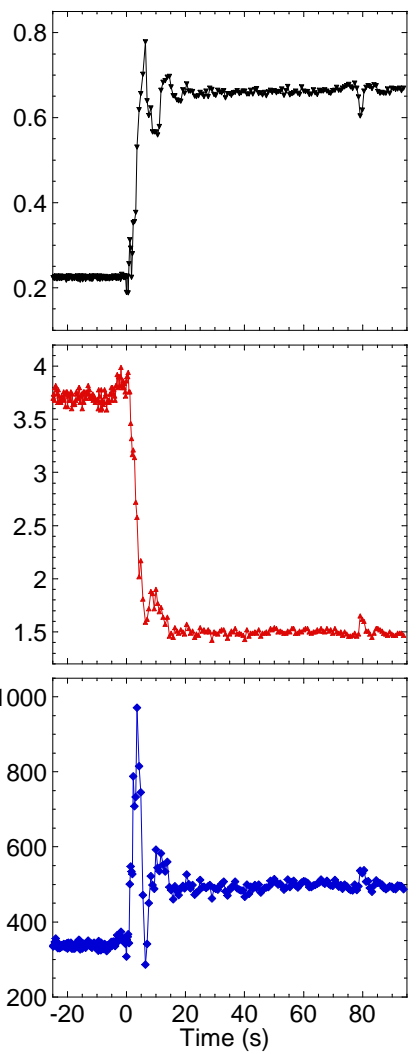

Fig. 6 Measured period of emission, velocity, and length, of droplets after a modification (at $t=0$ ) of the injection conditions: (a) increase and (b) decrease in flow rates, and (c) increase and $(\mathrm{d})$ decrease in pressures. Changes in flow rates $\left(Q_{o}, Q_{w}\right)$, expressed in $\mu \mathrm{L} \mathrm{min}^{-1}:$ (a) $(0.4,0.1) \rightarrow(25,2.5)$, (b) $(25,2.5) \rightarrow(0.4,0.1)$; changes in pressures $\left(\Delta p_{o}, \Delta p_{w}\right)$, expressed in Pa: $($ c) $(1566,1699) \rightarrow(1869,1699),(d)(3632,3392) \rightarrow(1964,2007)$.

Changes in imposed pressure are represented in Fig. 6 (c,d). Pressure changes are performed by manually lowering or raising the suspended reservoirs and therefore take several seconds. On the one hand, when increasing oil pressure solely [Fig. 6 (c)], the transition regime is quite steep. On the other hand, by decreasing both oil and water pressures [Fig. 6 (d)], one can observe a larger transient regime, that exhibits damped oscillations. The decay time of such oscillations is typically of the 
order of $20 \mathrm{~s}$. This time scale is comparable to that of pressure changes, suggesting that transient effect in pressure-driven flows are weaker than in flow-rate-driven flows. However, even though the transient regimes seem to be shorter in the pressure case, it must be kept in mind that the magnitude of the corresponding changes is much smaller. Indeed, pressure changed by several tens of percent, which has to be compared to a variation of more than one order of magnitude in flow rates - such a change in pressure is not easily reachable just by gravity.

The present basic study yields to the following conclusions. (i) Increasing or decreasing the imposed conditions (especially flow rates) do not lead to symmetric transient behaviors. (ii) The transient regimes are different in nature depending on the injection conditions: for a decrease of the flow rates, individual droplet characteristics change monotonically, while for a decrease of the pressures, characteristics exhibit damped oscillations. Such a distinction should be studied within the analogy between electric and fluidic circuits (Jousse et al 2005).

4.3 Effect of an external forcing: thermocapillary valve

The last example consists in evaluating the influence of an external forcing on the basic rate-driven flow. Several techniques, including fluid-structure interaction (Willaime et al 2006; Abate et al 2009; Galas et al 2009), or electrical control (Link et al 2006), have been developed for modifying in real time droplet individual characteristics. In this context, we have recently developed a laser-induced thermocapillary approach, which consists in temporary interrupting the water flow with a laser beam, yielding to larger droplets (Baroud et al 2007a,b). Regarding the natural fluctuations of droplet emission illustrated above, one can wonder if this external forcing method 


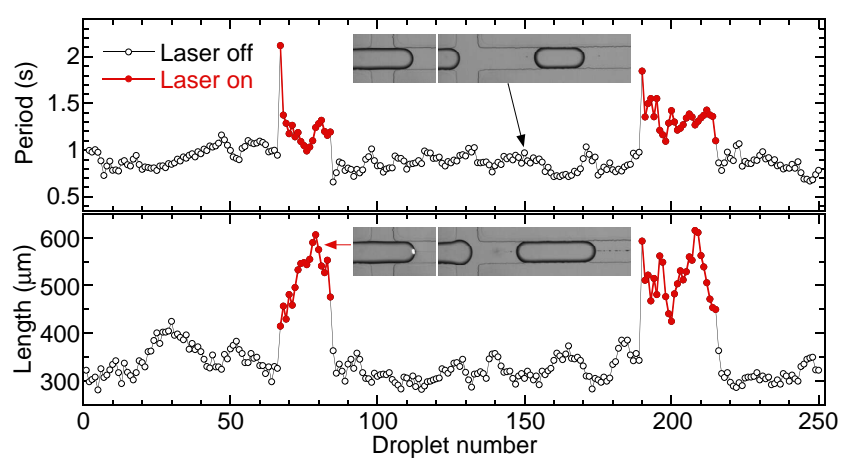

Fig. 7 Period of emission (top) and length (bottom) of successive drops. When the laser (white dot in the bottom left snapshot) is turned on, the front interface is transitorily blocked during droplet formation. Flow rates are $0.4 \mu \mathrm{L} \mathrm{min}^{-1}$ (oil) and $0.1 \mu \mathrm{L} \mathrm{min}^{-1}$ (water); laser power is $56 \mathrm{~mW}$, and beam waist is $5.2 \mu \mathrm{m}$.

provides significant changes in droplet characteristics. Figure 7 represents both period of emission and length of about 250 successive droplets, with (filled symbols) or without laser (open symbols). The two corresponding populations are clearly splitted. In spite of the natural dispersion, the external forcing clearly represents a possible means of controlling individual droplet properties.

\section{Conclusions}

In this paper, we presented a method for characterizing two-phase flows in microchannels, based on the differential detection of the variations in illumination on a pair of close photodiodes connected head-to-tail. This method is not limited by the size nor the optical properties of the detected droplets, and can therefore be used for studying a wide range of diphasic systems (cells, emulsions, polymeric liquids, etc.) as far as the channel is optically transparent. Furthermore, the complete disconnection between this device and the channel ensures its total portability. 
As reported in the present investigation, examples of microfluidic studies which can be performed include the characterization of stationary and transient regimes during very long times. Moreover, the real-time character of the measurement allows for using it for fast in situ experimental adjustments.

Since the detection is driven by the contrast in index of refraction, this detector might be a basis for analyzing the chemical composition of droplets, though another interpretation of the signal could be necessary. Applications to characterizations in the fields of analytical or synthetic chemistry can thus be envisioned.

Finally, since the detector reduces the drop shape to a length along the flow, one can wonder about consequences in terms of missing information compared to other characterization methods. Drop emission frequencies and velocity are not affected. The real cost is linked to the drop shape as far as the desired application requires droplet morphology investigation; in this case 2D video will be necessary. However, as shown in the present investigation, both methods are not exclusive and can be performed cooperatively.

Acknowledgements The authors gratefully acknowledge Rhodia's Laboratory of the Future (LoF) for access to microfabrication facilities, and Jean-Baptiste Salmon for technical advice. This work was partly supported by the CNRS and the Conseil régional d'Aquitaine through grant No. 20061102030

\section{References}

Abate AR, Romanowsky MB, Agresti JJ, Weitz DA (2009) Valve-based flow focusing for drop formation. Appl Phys Lett 94:023503, DOI 10.1063/1.3067862, URL http://link.aip.org/link/?APL/94/023503/1 
Baker CA, Duong CT, Grimley A, Roper MG (2009) Recent advances in microfluidic detection systems. Bioanalysis 1:967-975, URL http://www.futurescience.com/doi/abs/10.4155/bio.09.86

Baroud CN, Delville JP, Gallaire F, Wunenburger R (2007a) Thermocapillary valve for droplet production and sorting. Phys Rev E 75:046,302, DOI 10.1103/PhysRevE.75.046302

Baroud CN, Robert de Saint Vincent M, Delville JP (2007b) An optical toolbox for total control of droplet microfluidics. Lab Chip 7:1029-1033, URL http://www.rsc.org/Publishing/Journals/LC/article.asp?doi=b702472j

Bong KW, Chapin SC, Pregibon DC, Baah D, Floyd-Smith TM, Doyle PS (2011) Compressed-air flow control system. Lab Chip 11:743-747, DOI 10.1039/C0LC00303D, URL http://dx.doi.org/10.1039/C0LC00303D

Cartellier A (1990) Optical probes for local void fraction measurements: characterization of performance. Rev Sci Instrum 61:874-886, DOI 10.1063/1.1141457, URL http://link.aip.org/link/?RSI/61/874/1

Cartellier A (1992) Simultaneous void fraction measurement, bubble velocity, and size estimate using a single optical probe in gas-liquid two-phase flows. Rev Sci Instrum 63:5442-5453, DOI 10.1063/1.1143416, URL http://link.aip.org/link/RSINAK/v63/i11/p5442/s1

Christopher GF, Anna SL (2007) Microfluidic methods for generating continuous droplet streams. J Phys D: Appl Phys 40:R319-R336, URL http://stacks.iop.org/0022$3727 / 40 / \mathrm{i}=19 / \mathrm{a}=\mathrm{R} 01$

Cox JA, Zins CJ (2006) Cytometer cell counting and size measurement method. International Patent WO/2006/119106

Cox JA, Zins CJ (2010) Particle parameter determination system. US Patent 7688427

Dittrich PS, Tachikawa K, Manz A (2006) Micro total analysis systems. latest advancements and trends. Anal Chem 78:3887-3908, DOI 10.1021/, URL http://dx.doi.org/10.1021/ac0605602

Duffy DC, McDonald JC, Schueller OJA, Whitesides GM (1998) Rapid prototyping of microfluidic systems in poly(dimethylsiloxane). Anal Chem 70:4974-4984, URL http://dx.doi.org/10.1021/ac980656z 
Engl W, Roché M, Colin A, Panizza P, Ajdari A (2005) Droplet traffic at a simple junction at low capillary numbers. Phys Rev Lett 95:208,304, DOI 10.1103/PhysRevLett.95.208304, URL http://link.aps.org/doi/10.1103/PhysRevLett.95.208304

Galas JC, Bartolo D, Studer V (2009) Active connectors for microfluidic drops on demand. New J Phys 11:075,027, URL http://stacks.iop.org/1367-2630/11/i=7/a=075027

Götz S, Karst U (2007) Recent developments in optical detection methods for microchip separations. Anal Bioanal Chem 387:183-192, URL http://dx.doi.org/10.1007/s00216-006$0820-8$

Hironaga K, Nishiura T (1985) Apparatus for measuring two-phase flow. US Patent 4516432 Huebner A, Sharma S, Srisa-Art M, Hollfelder F, Edel JB, deMello AJ (2008) Microdroplets: A sea of applications? Lab Chip 8:1244-1254, DOI 10.1039/B806405A, URL http://dx.doi.org/10.1039/B806405A

Jakiela S, Makulska S, Korczyk PM, Garstecki P (2011) Speed of flow of individual droplets in microfluidic channels as a function of the capillary number, volume of droplets and contrast of viscosities. Lab Chip 11:3603-3608, DOI 10.1039/C1LC20534J, URL http://dx.doi.org/10.1039/C1LC20534J

Jousse F, Lian G, Janes R, Melrose J (2005) Compact model for multi-phase liquid-liquid flows in micro-fluidic devices. Lab Chip 5:646-656

Korczyk PM, Cybulski O, Makulska S, Garstecki P (2011) Effects of unsteadiness of the rates of flow on the dynamics of formation of droplets in microfluidic systems. Lab Chip 11:173175, DOI 10.1039/C0LC00088D, URL http://dx.doi.org/10.1039/C0LC00088D

de Lasa HI, Lee SL, Bergougnou MA (1987) Multi-probe system for measuring bubble characteristics gas hold-up, liquid hold-up and solid hold-up in a three-phase fluidized bed. US Patent 4659218

Lee W, Walker LM, Anna SL (2009) Role of geometry and fluid properties in droplet and thread formation processes in planar flow focusing. Phys Fluids 21:032103, DOI 10.1063/1.3081407, URL http://link.aip.org/link/?PHF/21/032103/1

Lim HJ, Chang KA, Su CB, Chen CY (2008) Bubble velocity, diameter, and void fraction measurements in a multiphase flow using fiber optic reflectometer. Rev Sci Instrum 79:125105, DOI 10.1063/1.3053271, URL http://link.aip.org/link/?RSI/79/125105/1 
Link DR, Grasland-Mongrain E, Duri A, Sarrazin F, Cheng Z, Cristobal G, Marquez M, Weitz DA (2006) Electric control of droplets in microfluidic devices. Angew Chem Int Ed 45:25562560, DOI 10.1002/anie.200503540, URL http://dx.doi.org/10.1002/anie.200503540

Liu S, Gu Y, Le Roux RB, Matthews SM, Bratton D, Yunus K, Fisher AC, Huck WTS (2008) The electrochemical detection of droplets in microfluidic devices. Lab Chip 8:1937-1942, DOI 10.1039/B809744E, URL http://dx.doi.org/10.1039/B809744E

Milne G, Rhodes D, MacDonald M, Dholakia K (2007) Fractionation of polydisperse colloid with acousto-optically generated potential energy landscapes. Opt Lett 32(9):1144-1146, DOI 10.1364/OL.32.001144, URL http://ol.osa.org/abstract.cfm?URI=ol-32-9-1144

Nguyen NT, Lassemono S, Chollet FA (2006) Optical detection for droplet size control in microfluidic droplet-based analysis systems. Sens Actuators B 117:431-436, DOI DOI: 10.1016/j.snb.2005.12.010, URL http://www.sciencedirect.com/science/article/B6THH4J2M4CB-9/2/1ca9ced8282d056b3c8a94d40a78f2ea

Niu X, Zhang M, Peng S, Wen W, Sheng P (2007) Real-time detection, control, and sorting of microfluidic droplets. Biomicrofluidics 1:044101, DOI 10.1063/1.2795392, URL http://link.aip.org/link/BIOMGB/v1/i4/p044101/s1

Padgett M, Di Leonardo R (2011) Holographic optical tweezers and their relevance to lab on chip devices. Lab Chip 11:1196-1205, DOI 10.1039/C0LC00526F, URL http://dx.doi.org/10.1039/C0LC00526F

Revellin R, Dupont V, Ursenbacher T, Thome JR, Zun I (2006) Characterization of diabatic two-phase flows in microchannels: Flow parameter results for r-134a in a $0.5 \mathrm{~mm}$ channel. Int J Multiphase Flow 32:755 - 774, DOI 10.1016/j.ijmultiphaseflow.2006.02.016, URL http://www.sciencedirect.com/science/article/pii/S0301932206000541

Song H, Chen DL, Ismagilov RF (2006) Reactions in droplets in microfluidic channels. Angew Chem Int Ed 45:7336-7356, URL http://dx.doi.org/10.1002/anie.200601554

Sun T, Morgan H (2010) Single-cell microfluidic impedance cytometry: a review. Microfluid Nanofluid 8:423-443, URL http://dx.doi.org/10.1007/s10404-010-0580-9

Teh SY, Lin R, Hung LH, Lee AP (2008) Droplet microfluidics. Lab Chip 8:198-220, DOI 10.1039/B715524G, URL http://dx.doi.org/10.1039/B715524G 
Trivedi V, Doshi A, Kurup GK, Ereifej E, Vandevord PJ, Basu AS (2010) A modular approach for the generation, storage, mixing, and detection of droplet libraries for high throughput screening. Lab Chip 10:2433-2442, DOI 10.1039/C004768F, URL http://dx.doi.org/10.1039/C004768F

Ward T, Faivre M, Abkarian M, Stone HA (2005) Microfluidic flow focusing: Drop size and scaling in pressure versus flow-rate-driven pumping. Electrophoresis 26:3716-3724, DOI 10.1002/elps.200500173, URL http://dx.doi.org/10.1002/elps.200500173

Willaime H, Barbier V, Kloul L, Maine S, Tabeling P (2006) Arnold tongues in a microfluidic drop emitter. Phys Rev Lett 96:054501, DOI 10.1103/PhysRevLett.96.054501, URL http://link.aps.org/abstract/PRL/v96/e054501 